\title{
Osteomielite maxilar aguda em paciente acometido por osteopetrose infantil maligna: relato de um caso raro de sobrevida
}

\author{
Acute maxillary osteomyelitis in a patient affected by malignant infantile \\ osteopetrosis: report of a rare case of survival
}

\author{
Bruno Limaverde Vilar Lobo', Natália Freitas Francelino Dias², Mércia Lima de Carvalho \\ Lemos $^{3}$, Lia Cavalcanti de Albuquerque ${ }^{4}$
}

Lobo BLV, Dias NFF, Lemos MLC, Albuquerque LC. Osteomielite maxilar aguda em paciente acometido por osteopetrose infantil maligna: relato de um caso raro de sobrevida / Acute maxillary osteomyelitis in a patient affected by malignant infantile osteopetrosis: report of a rare case of survival. Rev Med (São Paulo). 2020 jul.-ago.;99(4):405-10.

\begin{abstract}
RESUMO: A osteopetrose infantil maligna (OIM) é a forma mais grave de osteopetrose (OP), que é um grupo de desordens raras e hereditárias que acometem o esqueleto humano, tornando-o mais denso. Uma das suas principais consequências é a invasão medular por esclerose óssea, levando a progressiva insuficiência medular e aplasia, o que predispõe a inúmeras infecções graves, incluindo a osteomielite. É uma doença que possui mortalidade de $99 \%$ até os dez anos de idade, com incidência de 1:500.000 nascidos vivos, cujo único tratamento disponível é o transplante de medula óssea precoce. $\mathrm{O}$ caso relatado é de um paciente do sexo masculino, 14 anos, com diagnóstico de OIM desde a infância, admitido na emergência do Hospital Infantil Albert Sabin com edema e dor facial à direita de início há um mês. Com base no exame físico e nos exames complementares foi diagnosticada osteomielite maxilar aguda. Além do quadro atual, o paciente possuía diversas sequelas da sua doença de base. Evoluiu com sepse durante a internação, fez uso de antibioticoterapia, e obteve alta hospitalar após um mês para cuidados paliativos domiciliares.
\end{abstract}

Descritores: Osteopetrose/complicações; Sobrevida; Osteomielite; Adolescente.

\begin{abstract}
Infantile malignant osteopetrosis (IMO) is the most severe form of osteopetrosis (OP), which is a group of rare and hereditary disorders that affect the human skeleton by increasing bone density. One of its main consequences is bone marrow invasion by bone sclerosis, leading to progressive bone marrow failure and aplasia, which predisposes to several infections, including osteomyelitis. It is a disease with $99 \%$ mortality by the age of ten and an incidence of 1:500,000 live births. The only available treatment is early bone marrow transplantation. The case report describes a 14-year old male patient, diagnosed with IMO since childhood and admitted to the emergency room of the Albert Sabin Children's Hospital with right facial edema and local pain that started one month before admission. Acute osteomyelitis of the maxilla was diagnosed based on physical examination and complementary tests. In addition to the current condition, the patient had several complications from his underlying disease. He developed sepsis during hospitalization, used antibiotic therapy, and was discharged after one month for home palliative care.
\end{abstract}

Keywords: Osteopetrosis/complications; Survival; Osteomyelitis; Adolescent.

\footnotetext{
Trabalho realizado no Hospital Infantil Albert Sabin.

1. Médico Residente de Medicina da Família e Comunidade da Escola de Saúde Pública do Ceará, Fortaleza, CE. ORCID: https//orcid.org/0000-00019368-5341. Email: blvlobo@gmail.com.

2. Médica Residente de Medicina Intensiva Pediátrica do Instituto de Medicina Integral Professor Fernando Figueira (IMIP), Recife, PE, ORCID: https// orcid.org/0000-0003-2446-431. Email: nataliaffdias@hotmail.com.

3. Médica Pediatra Assistente do Hospital Infantil Albert Sabin (HIAS), Fortaleza, CE, ORCID: https//orcid.org/0000-0001-6177-784X. Email: mercia.1@ hotmail.com.

4. Médica Pediatra Assistente do Hospital Infantil Albert Sabin (HIAS), professora de práticas médicas do curso de medicina da Universidade Estadual do Ceará, Fortaleza, CE, ORCID: https//orcid.org/0000-0001-5123-0525. Email: lia_cavalcanti@yahoo.com.br.

Endereço para correspondência: Bruno Limaverde Vilar Lobo. Rua Haroldo Torres, n $^{\circ}$ 1111, ap. 206. bairro São Gerardo, Fortaleza, Ceará, Brasil. CEP: 60320-104. Email: blvlobo@gmail.com.
} 


\section{INTRODUÇÃO}

steopetrose (OP) é uma denominação para um
grupo de desordens raras e hereditárias que acometem o esqueleto humano, tornando-o mais denso ${ }^{1}$. Mutações em mais de 12 genes já foram identificadas como responsáveis pela causa dessas desordens, sendo mais comumente encontradas em filhos de pais consanguíneos ${ }^{2,3}$.

A incidência mundial exata da OP é desconhecida, mas acredita-se que, a de sua forma maligna, seja em torno de uma pessoa acometida em cada 100.000 a 500.000 da população geral. A doença tem um amplo espectro de apresentação e pode ser classificada em três tipos principais: OP infantil maligna, OP intermediária e OP de início tardio ${ }^{4}$. A OP infantil maligna é a apresentação mais grave da doença e costuma iniciar seus sintomas de pancitopenia e de esplenomegalia dos primeiros meses aos primeiros anos de vida ${ }^{5}$. A sobrevida dessas crianças é bastante reduzida. Cerca de $70 \%$ delas vão a óbito com menos de seis anos, e até $99 \%$ com menos de 10 anos de idade $^{6}$, sendo o transplante de medula óssea precoce o único tratamento curativo possível ${ }^{7}$.

A principal causa de internação hospitalar e de morte pela doença é a progressiva falência medular, causada pela esclerose óssea que invade a medula, levando à aplasia ${ }^{8}$. Assim, o óbito na primeira década de vida geralmente se dá por infecções secundárias à neutropenia, por hemorragias secundárias à trombocitopenia ou por anemia grave ${ }^{9}$.

Além de diversas complicações hematológicas e neurológicas da doença, uma infecção comumente associada à OP é a osteomielite facial, que acomete principalmente a região da mandíbula e, mais raramente, da maxila nesses pacientes. Geralmente ocorre devido a extração dentária ou ao mau estado de conservação dos dentes, causando grande morbidade ${ }^{10}$.

Este artigo se propõe a relatar o caso de um adolescente com 14 anos de idade, portador de osteopetrose infantil maligna, com diversas complicações da doença, dentre elas um quadro agudo de osteomielite maxilar.

\section{RELATO}

Adolescente, 14 anos, sexo masculino, natural do interior do Ceará, filho de pais consanguíneos, acompanhado no ambulatório de onco-hematologia do Hospital Infantil Albert Sabin (HIAS), por falência medular secundária à osteopetrose infantil maligna (OIM). Chegou ao setor de emergência do HIAS com história de que, há um mês do internamento, iniciou quadro de edema facial à direita com dor local associada, porém sem febre. Procurou atendimento médico em sua cidade onde foi prescrito antibiótico via oral. Há dez dias do internamento houve piora dos sintomas, fazendo-os procurar assistência em Fortaleza, no HIAS.

A história pregressa revelou que o paciente apresentava anemia desde os dois meses de idade, sendo diagnosticado com OIM tardiamente, por volta dos quatro anos de idade. Os pais relatam que tiveram outro filho que foi a óbito com poucos anos de vida devido à mesma doença. O paciente teve internações prévias por complicações secundárias à insuficiência medular. Em 2012, realizou enucleação ocular direita, necessitando de traqueostomia por dificuldade de intubação orotraqueal durante o procedimento cirúrgico. Em relação aos hábitos de vida, a mãe referiu dificuldade na higienização da cavidade oral do filho.

$\mathrm{Na}$ admissão, o paciente se encontrava hipocorado (2/4+), afebril, ativo, macrocefálico, cego, com hipoacusia bilateral, taquipneico, taquicárdico, traqueostomizado, com edema facial à direita em região maxilar (Figura 1A), apresentando sinais flogísticos, com cicatriz de enucleação ocular à direita e exoftalmia à esquerda. Ausculta cardiopulmonar sem alterações. Abdome globoso, de consistência endurecida, com hepatoesplenomegalia volumosa, palpáveis abaixo da cicatriz umbilical (Figura 1B).

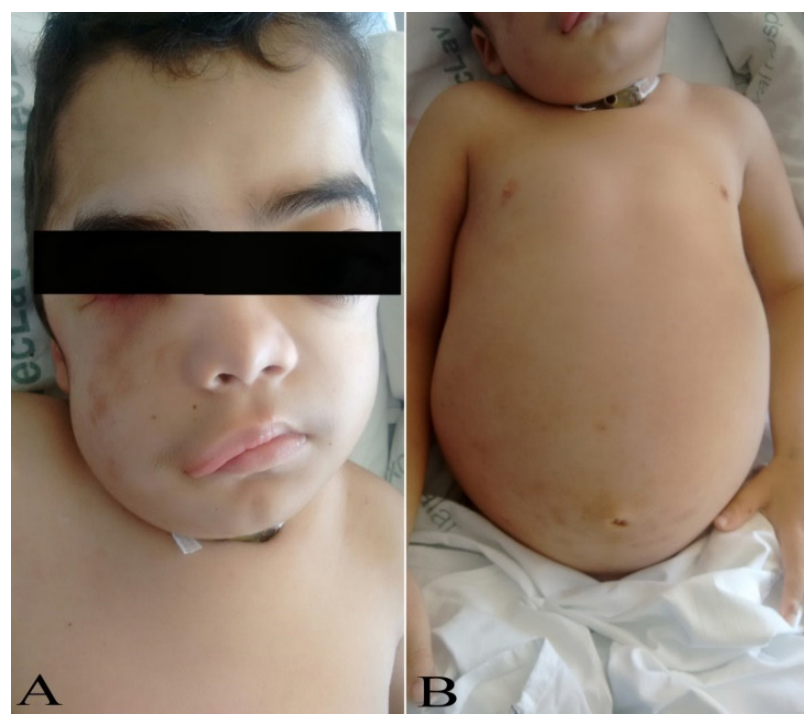

Figura 1 - Achados ao exame físico. A) Edema facial à direita em região maxilar. B) Abdome globoso, endurecido, com hepatoesplenomegalia volumosa, palpável até fossa ilíaca direita

Os exames laboratoriais evidenciaram anemia (hemoglobina de $8,1 \mathrm{~g} / \mathrm{dL}$ ), plaquetopenia (35.390 plaquetas $/ \mathrm{mm}^{3}$ ), lactato desidrogenase (LDH) de 1071 U/L, fosfatase alcalina de $131 \mathrm{U} / \mathrm{L}$ com função renal e eletrólitos normais (Tabela 1). Não foi possível visualizar com precisão o parênquima pulmonar no raio $\mathrm{X}$ de tórax devido à grande densidade óssea das costelas (Figura 2). 


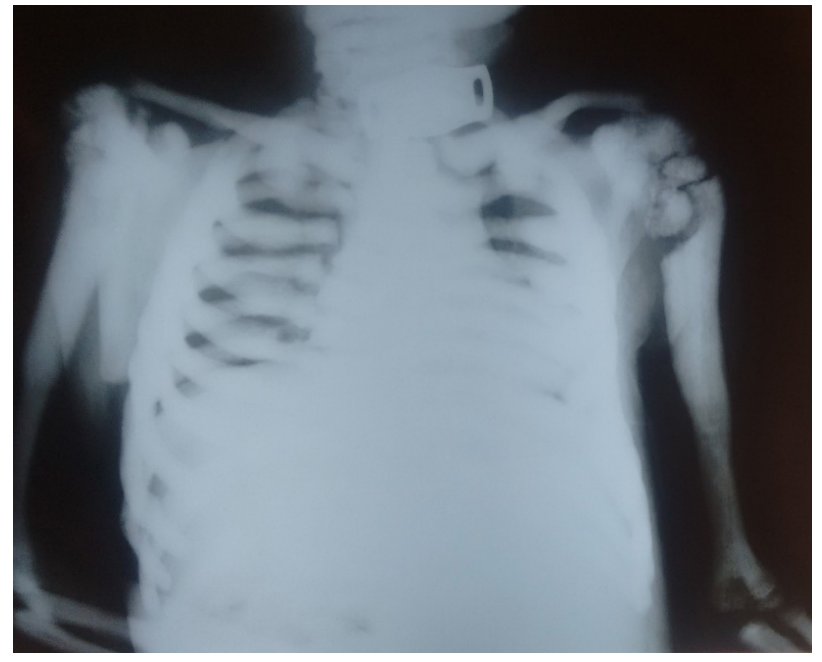

Figura 2 - Raio X de tórax evidenciando acentuada esclerose óssea nas costelas

Tabela 1 - Exames laboratoriais do período de internação
Decidiu-se iniciar antibioticoterapia intravenosa com oxacilina e ceftriaxona e internar o paciente na enfermaria de pediatria geral. Ele evoluiu dois dias depois com pancitopenia (Hb: 6,6 g/dL; 3394 leucócitos $/ \mathrm{mm}^{3}$ e 27.390 plaquetas $/ \mathrm{mm}^{3}$ ), além de sangramento pelo traqueóstomo e epistaxe, sendo realizada transfusão de hemácias e plaquetas. A tomografia de crânio com contraste mostrou edema subcutâneo em face direita e grande esclerose dos ossos da face pela atividade da doença de base, não sendo possível definir área de osteomielite (Figura 3). Foi feita dexametasona por sete dias com melhora parcial do edema e da dor.

A equipe de cirurgia bucomaxilar do hospital confirmou o diagnóstico de osteomielite maxilar ao visualizar a cavidade bucal com processo inflamatório, gerando porta de entrada infecciosa ao lado de erupção dentária deficiente em maxila direita (Figura 4). A conduta proposta foi continuar antibioticoterapia e higienizar a cavidade oral, pois procedimentos cirúrgicos estavam contraindicados pelo alto risco de hemorragia.

\begin{tabular}{|c|c|c|c|c|}
\hline Exames & $1^{\circ}$ dia de internação & $12^{\circ}$ dia de internação & $20^{\circ}$ dia de internação & Referência \\
\hline Hemoglobina & $8,1 \mathrm{~g} / \mathrm{dL}^{*}$ & $7,3 \mathrm{~g} / \mathrm{dL}^{*}$ & $8,2 \mathrm{~g} / \mathrm{dL}^{*}$ & $12-16 \mathrm{~g} / \mathrm{dL}$ \\
\hline Leucócitos & $5.630 / \mathrm{mm}^{3 *}$ & $2.660 / \mathrm{mm}^{3 *}$ & $4.312 / \mathrm{mm}^{3 *}$ & $4.500-13.500 / \mathrm{mm}^{3}$ \\
\hline Neutrófilos & $2.983 / \mathrm{mm}^{3 *}$ & $1.197 / \mathrm{mm}^{3 *}$ & $2.630 / \mathrm{mm}^{3 *}$ & $4.300-5.300 / \mathrm{mm}^{3}$ \\
\hline Plaquetas & $35.390 / \mathrm{mm}^{3 *}$ & $20.440 / \mathrm{mm}^{3 *}$ & $34.130 / \mathrm{mm}^{3 *}$ & $150.000-440.000 / \mathrm{mm}^{3}$ \\
\hline Reticulócitos & $5,56 \% *$ & - & - & $0,5-2,5 \%$ \\
\hline Albumina & $3,4 \mathrm{~g} / \mathrm{dL}^{*}$ & - & - & $3,5-5 \mathrm{~g} / \mathrm{dL}$ \\
\hline Proteína $\mathrm{C}$ reativa & & $192 \mathrm{mg} / \mathrm{dL}^{*}$ & - & $<10 \mathrm{mg} / \mathrm{dL}$ \\
\hline Sódio & $139 \mathrm{mEq} / \mathrm{L}$ & - & $123 \mathrm{mEq} / \mathrm{L}^{*}$ & $135-145 \mathrm{mEq} / \mathrm{L}$ \\
\hline Potássio & $3,8 \mathrm{mEq} / \mathrm{L}$ & - & $3,7 \mathrm{mEq} / \mathrm{L}$ & $3,5-5,5 \mathrm{mEq} / \mathrm{L}$ \\
\hline Cálcio & $9,25 \mathrm{mg} / \mathrm{dL}$ & - & $6,25 \mathrm{mg} / \mathrm{dL}^{*}$ & $8,5-10,5 \mathrm{mg} / \mathrm{dL}$ \\
\hline Magnésio & $1,8 \mathrm{mg} / \mathrm{dL}^{*}$ & - & $1,1 \mathrm{mg} / \mathrm{dL}^{*}$ & $1,9-2,5 \mathrm{mg} / \mathrm{dL}$ \\
\hline Cloro & - & - & $88 \mathrm{mEq} / \mathrm{L}^{*}$ & $97-106 \mathrm{mEq} / \mathrm{L}$ \\
\hline Fósforo & $5,4 \mathrm{mg} / \mathrm{dL}$ & - & $2,7 \mathrm{mg} / \mathrm{dL}$ & $2,5-5,6 \mathrm{mg} / \mathrm{dL}$ \\
\hline Fosfatase alcalina & $131 \mathrm{U} / \mathrm{L}$ & $135 \mathrm{U} / \mathrm{L}$ & - & $65-300 \mathrm{U} / \mathrm{L}$ \\
\hline LDH & $1071 \mathrm{U} / \mathrm{L}^{*}$ & $1177 \mathrm{U} / \mathrm{L}^{*}$ & $1019 \mathrm{U} / \mathrm{L}^{*}$ & $230-450 \mathrm{U} / \mathrm{L}$ \\
\hline Creatinina sérica & $0,2 \mathrm{mg} / \mathrm{dL}^{*}$ & - & $0,2 \mathrm{mg} / \mathrm{dL}^{*}$ & $0,6-1,3 \mathrm{mg} / \mathrm{dL}$ \\
\hline Ureia & $27 \mathrm{mg} / \mathrm{dL}$ & - & - & $5-40 \mathrm{mg} / \mathrm{dL}$ \\
\hline
\end{tabular}

Legenda: *: Exames alterados; LDH: lactato desidrogenase 


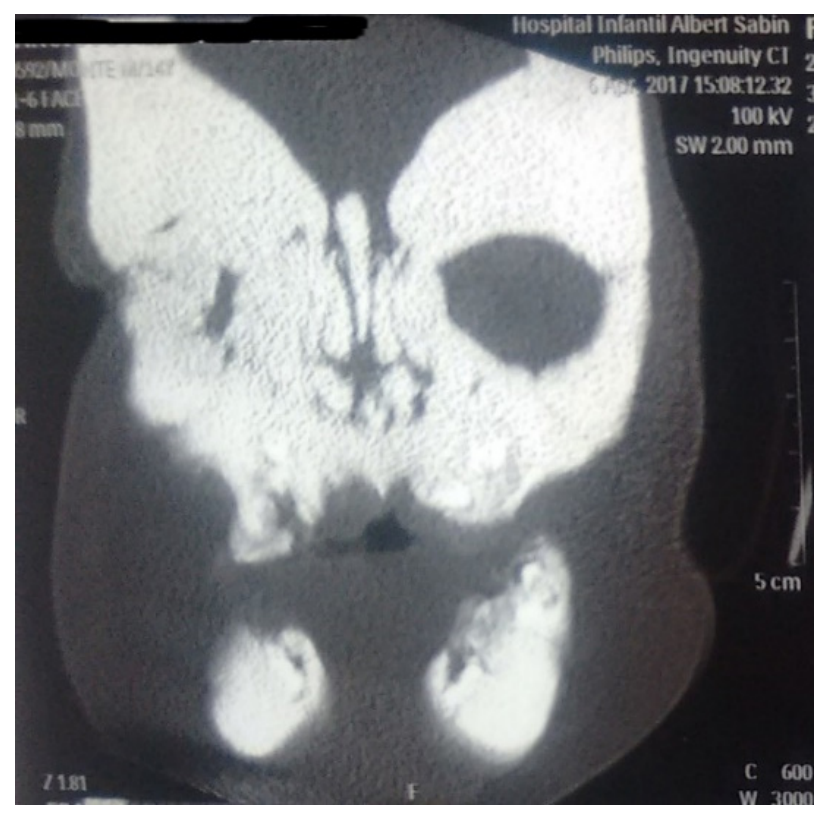

Figura 3 - Corte de tomografia de face com contraste evidenciando edema subcutâneo a direita e esclerose óssea da face

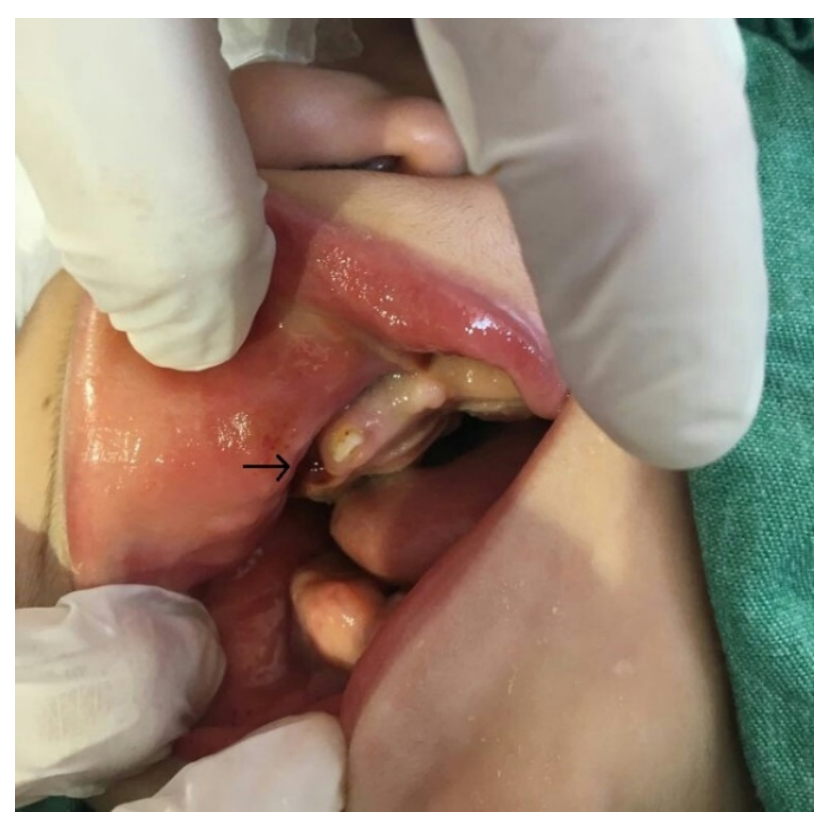

Figura 4 - Imagem evidenciando ausência de erupção dentária em quase toda a cavidade oral. Seta mostrando provável porta de entrada da infecção, lateralmente à erupção dentária deficiente, em maxila direita

Por volta do décimo segundo dia de internação o paciente começou a apresentar diarreia, episódios de febre diária, taquipneia, taquicardia, piora do estado geral, dessaturação, necessitando de oxigênio suplementar em máscara de Venturi a 35\%, e tornando-se sonolento e hipoativo no leito. Foi, então, ampliado o espectro antimicrobiano com vancomicina e cefepime. Devido à plaquetopenia persistente, o sangramento pelo traqueóstomo e a epistaxe se tornaram mais frequentes, porém de pequena monta, sendo orientado pelo serviço de onco-hematologia fazer transfusão somente se os sangramentos ocorressem em grande volume.

A família foi comunicada sobre as condições clínicas do paciente, sua doença de base e prognóstico, tendo solicitado a não transferência para a UTI. A evolução seguiu com piora laboratorial e distúrbios hidroeletrolíticos, persistindo com LDH aumentado (1019 U/L), sódio sérico de $123 \mathrm{mEq} / \mathrm{L}$, cálcio de $6,2 \mathrm{mg} / \mathrm{dL}$, cloro de $88 \mathrm{mEq} / \mathrm{L}$ e magnésio de 1,1 mg/dL (Tabela 1 ). Foi, então, encaminhado para uma enfermaria de isolamento do Centro Pediátrico do Câncer (CPC), onde o paciente teria maior suporte de assistência paliativa. Com aproximadamente um mês de internação, em antibioticoterapia de amplo espectro, o paciente apresentou melhora do quadro séptico, recebendo alta do CPC para ser acompanhado ambulatorialmente em cuidados paliativos.

\section{DISCUSSÃO}

A osteopetrose é uma doença hereditária rara que possui um amplo espectro de apresentação, desde a forma benigna, autossômica dominante, com manifestações somente na fase adulta, até a forma infantil maligna, de herança autossômica recessiva, com alto índice de morbimortalidade ${ }^{4}$.

O caso relatado trata de um adolescente de 14 anos portador da forma maligna da doença, tipo mais grave, cuja incidência mundial gira em torno de um paciente para cada 250.000 nascidos vivos, valor que pode variar na literatura até um para 500.000 nascidos vivos ${ }^{4,11}$. Essa forma costuma iniciar seus sintomas nos primeiros meses após o nascimento, com anemia e esplenomegalia. $\mathrm{O}$ paciente em questão apresenta uma sobrevida acima da esperada, já que, segundo os relatos científicos prévios, esses pacientes têm uma expectativa de vida curta, com $99 \%$ deles não sobrevivendo aos dez anos de idade ${ }^{5,6}$. Os pais do adolescente possuíam dois importantes fatores de risco bastante citados na literatura: eram primos legítimos e já haviam tido outro filho que foi a óbito com poucos anos de vida, portador da mesma enfermidade ${ }^{12}$.

$\mathrm{O}$ paciente chegou ao Hospital Infantil Albert Sabin (HIAS) com queixa de edema facial à direita, sinais flogísticos e dor local. Ao exame físico da cavidade oral foi encontrado um foco inflamatório e infeccioso em maxila direita, sem acometimento de mandíbula. Esse achado corroborou o diagnóstico de uma das principais complicações agudas da osteopetrose que é a osteomielite de mandíbula ou, menos frequentemente, de maxila, como evidencia uma revisão de 47 casos de osteomielite facial, em pacientes com osteopetrose, a qual encontrou que apenas $27,7 \%$ dos casos se restringiam à maxila ${ }^{13}$.

Os principais fatores de risco, nesse contexto, são 
a extração dentária ou o mau estado de conservação dos dentes. Como há uma diminuição da produção dos leucócitos e uma deficiência vascular nos ossos, os pacientes com OP tendem a desenvolver processos infecciosos no tecido ósseo podendo evoluir com osteonecrose $\mathrm{e}^{10}$.

O adolescente possuía pobre erupção dentária e nunca havia sido submetido à extração de dentes, porém sua mãe relatou má higienização da cavidade bucal devido ao filho não ser colaborativo com esse procedimento, o que de fato pode ter predisposto à osteomielite.

A tomografia de face com contraste não contribuiu para o diagnóstico, devido à intensa esclerose óssea que dificultou a delimitação da área de osteomielite.

$\mathrm{O}$ agente etiológico mais comumente encontrado na osteomielite maxilar é o Staphylococcus aureus, seguido pelo Streptococcus do grupo hemolítico, Pneumococcus e algumas bactérias gram-negativas. Porém, o tratamento inicial com oxacilina e ceftriaxona não proporcionou melhora clínica no caso relatado, havendo necessidade de ampliar o espectro antimicrobiano com vancomicina e cefepime. É importante lembrar que a antibioticoterapia nesses pacientes não tem bons índices de cura, pois a densificação óssea dificulta a penetração das substâncias no local de infecção. Assim, o melhor tratamento seria a remoção cirúrgica do foco infeccioso ${ }^{14}$.

Como o paciente possuía dados laboratoriais compatíveis com doença avançada, isto é, com contagem de plaquetas chegando a menos de 30.000 por $\mathrm{mm}^{3}$, e com presença de epistaxe e sangramento pelo traqueóstomo, a equipe de cirurgia bucomaxilar do HIAS sugeriu a manutenção da antibioticoterapia intravenosa, apesar de não ser o tratamento padrão, e contraindicou qualquer intervenção cirúrgica ${ }^{15}$.

Além da predisposição à osteomielite, muitas complicações crônicas são típicas da doença ${ }^{16}$. O fator chave da osteopetrose é a ausência de atividade dos osteoclastos (células responsáveis pela reabsorção óssea), que se encontram inativados por mutações genéticas. Como os osteoblastos continuam desempenhando seu papel fisiológico, ocorre um crescimento exagerado da massa óssea e consequente esclerose dos ossos do corpo, levando aos vários sinais e sintomas encontrados na doença ${ }^{17}$.

A principal consequência da densificação óssea é a falência medular, que leva o paciente a ter pancitopenia e a necessitar de hematopoiese extramedular, com posterior desenvolvimento de hepatoesplenomegalia ${ }^{15}$. Em fase mais avançada, o indivíduo chega a ter aplasia da medula óssea, e o óbito na primeira década de vida geralmente se dá por infecções secundárias à neutropenia, por sangramentos secundários à trombocitopenia ou por anemia grave 9 .

No exame físico, vários achados característicos da doença foram compatíveis com os relatados em outros artigos: macrocefalia, exoftalmia, enucleação ocular, traqueostomia por dificuldade de intubação orotraqueal e hepatoesplenomegalia volumosa ${ }^{18}$. Esses pacientes também podem ter fragilidade óssea paradoxal, aumentando a predisposição a fraturas, estenose de coanas, dificuldade respiratória e dificuldades para engolir alimentos ${ }^{19}$. Manifestações neurológicas também são comuns nesse contexto devido à obstrução dos forames do crânio pelos quais os nervos cranianos e alguns importantes vasos sanguíneos passam ${ }^{20}$. Cegueira, surdez, paralisia facial e hidrocefalia são algumas dessas manifestações ${ }^{1}$. O paciente em questão era cego e apresentava hipoacusia bilateral.

O diagnóstico da OP é principalmente radiológico e laboratorial ${ }^{16}$. Nas imagens, encontra-se esclerose generalizada dos ossos, como evidenciado no raio $\mathrm{X}$ de tórax e na tomografia de face do paciente, além de espessamento do calvário, aumento da densidade medular, levando à aparência de "osso dentro do osso", dentre outras alterações radiológicas ${ }^{11}$.

Outra característica típica do diagnóstico da OP são os achados laboratoriais de pancitopenia, LDH e fosfatase alcalina aumentadas, hipocalcemia e possível redução das imunoglobulinas $^{19}$. O paciente chegou a apresentar LDH de $1071 \mathrm{U} / \mathrm{L}$, pancitopenia grave, com plaquetas de 27.390 por $\mathrm{mm}^{3}$ e hipocalcemia de $6,2 \mathrm{mg} / \mathrm{dL}$, ocasionada por déficit na reabsorção óssea ${ }^{11}$.

Em relação ao tratamento da OIM, a única terapia capaz de mudar o curso da doença é o transplante de medula óssea (TMO), o qual pode aumentar a sobrevida dos pacientes, desde que seja feito de forma bastante precoce e por meio de um doador o mais compatível possível, de preferência HLA idêntico ${ }^{7,20}$. Mesmo assim, o TMO pode trazer diversos riscos aos pacientes, e grande proporção deles ainda desenvolvem sequelas da doença apesar do tratamento ${ }^{21}$. O paciente, apesar de ter apresentado anemia desde os dois meses de idade, teve o diagnóstico tardio de OP, devido aos problemas socioeconômicos comuns à grande número de brasileiros. A vida no interior muitas vezes dificulta o acesso aos centros terciários de saúde, que concentram a grande maioria dos especialistas. Por isso, este relato também tem como objetivo alertar os pediatras e médicos generalistas para a existência dessa doença rara e para a necessidade de se fazer o diagnóstico precoce.

\section{CONCLUSÃo}

Apesar de rara, a OIM possui uma relevância científica muito alta, por se tratar de uma doença significativamente fatal e geradora de grande morbidade, além de desgaste físico e emocional para os familiares. Com o caso relatado, pôde-se verificar a manifestação de uma importante complicação aguda, osteomielite maxilar, causada pela má higienização dental em um paciente possuidor de grande predisposição a esse tipo de infecção. Faz-se necessária, portanto, a divulgação de tal doença rara, para que seu diagnóstico seja feito de forma precoce e suas complicações sejam evitadas. 
Participação dos autores: Lobo $B L V$ : contribuiu na concepção, delineamento, pesquisa, análise e interpretação dos dados e redação do manuscrito; Dias NFF: contribuiu na pesquisa, análise e interpretação dos dados, confecção das imagens e redação do manuscrito; Lemos MLC: contribuiu no delineamento, revisão crítica e aprovação final do manuscrito; Albuquerque LC: contribuiu no delineamento, revisão crítica e aprovação final do manuscrito.

\section{REFERENCIAS}

1. Kulkarni GB, Pal PK, Shyambabu C, Kovoor JME, Senthilkumar E. Osteopetrosis manifesting as recurrent bilateral facial palsy in childhood: a case report. Clin Neurol Neurosurg. 2011;113(3):230-34. doi: http://dx.doi. org/10.1016/j.clineuro.2010.09.015.

2. Moore JB, Hoang TD, Shwayhat AF. Case report of clinical vignette: osteopetrosis. Mil Med. 2017;182(3):1886-88. doi: http://dx.doi.org/10.7205/MILMED-D-16-00234.

3. Palagano E, Susani L, Menale C, Ramenghi U, Berger M, Uva P, Oppo M, Vezzoni P, Villa A, Sobacchi C. Genetics of osteopetrosis. Curr Osteoporos Rep. 2018;16(1):13-25. doi: http://dx.doi.org/10.1007/s11914-018-0415-2.

4. Infante-Cossio P, Perez LMG, Fuentes RM, InfanteCossio M, Seiquer AC, Castellanos EJ. Maxillomandibular osteomyelitis associated with osteopetrosis. J Craniofac Surg. 2014;25(1):2012-15. doi: http://dx.doi.org/10.1097/ SCS.0000000000000422.

5. Machado CV, Da Rocha MCBS, Telles PDS. Infantile Osteopetrosis associated with osteomyelitis. BMJ Case Rep. 2015;2015:1-5. doi: http://dx.doi.org/10.1136/bcr-2014208085.

6. Jenkins PF, Prieto P, Tang RA, Yousefi S. Osteopetrosis. Am Orthopt J. 2013;63(1):107-11. doi: http://dx.doi.org/10.3368/ aoj.63.1.107.

7. Taheri APH, Radmard AR, Kooraki S, Behfar M, Pak N, Hamidieh AA, Ghavamzadeh A. Radiologic resolution of malignant infantile osteopetrosis skeletal changes following hematopoietic stem cell transplantation. Pediatr Blood Cancer. 2015;53:1645-49. doi: http://dx.doi.org/10.1002/pbc.25524.

8. Trivellato AE, Ribeiro MC, Sverzut CE, Bonuccu E, Nanci A, Oliveira PT. Osteopetrosis complicated by osteomyelitis of the maxilla and mandible: Light and electron microscopic findings. Head Neck Pathol. 2009;3(4):320-26. doi: http:// dx.doi.org/10.1007/s12105-009-0142-1.

9. Natsheh J, Drozdinsky G, Simanovsky N, Lamdan R, Erlich O, Gorelik N, Or R, Weintraub M, Stepensky P. Improved outcomes of hematopoietic stem cell transplantation in patients with infantile malignant osteopetrosis using fludarabine-based conditioning. Pediatr Blood Cancer. 2016;63:535-40. doi: http://dx.doi.org/10.1002/pbc.25801.

10. Yamada T, Mishima K, Imura H, Ueno T, Matsumura T, Moritani N, Sugahari T. Osteomyelitis of the mandible secondary to infantile osteopetrosis: a case report. Oral Surg Oral Med Oral Pathol Oral Radiol Endod. 2009;107(6):25-29. doi: http://dx.doi.org/10.1016/j.tripleo.2009.02.015.

11. Simanovsky N, Rozovsky K, Hiller N, Weintraub M,
Stepensky P. Extending the spectrum of radiological findings in patients with severe osteopetrosis and different genetic backgrounds. Pediatr Blood Cancer. 2016;53:1222-26. doi: http://dx.doi.org/10.1002/pbc.25952.

12. Shadur B, Zaidman I, Eddin AN, Lokshin E, Hussein Fatma, Oron HC, Avni B, Grisariu S, Stepensky P. Successful hematopoietic stem cell transplantation for osteopetrosis using reduced intensity conditioning. Pediatr Blood Cancer. 2018;65(6):1-7. doi: http://dx.doi.org/10.1002/pbc.27010.

13. Mikami T, Miake Y, Molina RB, Takeda Y. Ultrastructural analyses of alveolar bone in a patient with osteomyelitis secondary to osteopetrosis: a review of the literature. J Oral Maxilofac Surg. 2016;74(8):1584-95. doi: http://dx.doi. org/10.1016/j.joms.2016.02.016.

14. Sun HJ, Xue MS, Lei; Wu CB, Zhou Q. Clinical characteristics and treatment of osteopetrosis complicated by osteomyelitis of the mandible. J Craniofac Surg. 2016;27(8);728-30. doi: http://dx.doi.org/10.1097/SCS.0000000000003048.

15. Yadav S, Chalise S, Chaudhary S, Shah GS, Grupta MK, Mishra OP. Osteopetrosis in two siblings: two case reports genetics. BMC Res Notes. 2016;9(1):3-6. doi: http://dx.doi. org/10.1186/s13104-016-1869-X.

16. Ogino Y, Ayukawa Y, Tomita Y, Koyano K. Prosthetic aspects in adult osteopetrosis. J Prosthet Dent. 2014;112(4):736-40. doi: http://dx.doi.org/10.1016/j.prosdent.2014.04.007.

17. Mehta M, Pushker N, Sethi S, Bajaj MS, Sharma S, Rajesh R, Ghose S. Oculo-orbital manifestations of osteopetrosis in an Indian patient. Ann Trop Med Parasitol. 2010;104(3);275-81. doi: http://dx.doi.org/10.1179/136485910X12647085215697.

18. Badv RS, Dehghani SS, Behfar M, Ahad B, Tabasi A, Hamidieh AA. Impact of hematopoietic stem cell transplant on VEP and ABR values of the patients with malignant infantile osteopetrosis. Eur J Paediatr Neurol. 2018;22(4);718-24. doi: http://dx.doi.org/10.1016/j.ejpn.2018.04.005.

19. Sobacchi C, Schluz A, Coxon FP, Villa A, Helfrich MH. Osteopetrosis: genetics, treatment and new insights into osteoclast function. Nat Rev Endocrinol. 2013;9(9);522-36. doi: http://dx.doi.org/10.1038/nrendo.2013.137.

20. Essabar L, Meskini T, Ettair S, Erreimi N, Mouane N. Malignant infantile osteopetrosis: case report with review of literature. Pan Afr Med J. 2014;17:1-7. doi: http://dx.doi. org/10.11604/pamj.2014.17.63.3759.

21. Steward CG. Hematopoietic stem cell transplantation for osteopetrosis. Pediatr Clin North Am. 2010;57(1):171-80. doi: http://dx.doi.org/10.1016/j.pcl.2009.11.006.

Recebido: 28/07/2019

Aceito: 28/05/2020 